\title{
Atmospheric scintillation at Dome C, Antarctica
}

\author{
Suzanne L. Kenyon ${ }^{1}$, Jon S. Lawrence ${ }^{1}$, Michael C. B. Ashley ${ }^{1}$, \\ John W. V. Storey ${ }^{1}$, Andrei Tokovinin ${ }^{2}$ and Eric Fossat ${ }^{3}$ \\ ${ }^{1}$ School of Physics, University of New South Wales, Sydney, NSW 2052, Australia \\ email: suzanne@phys.unsw.edu.au \\ ${ }^{2}$ Laboratoire Universitaire d'Astrophysique de Nice, UMR 6525, \\ Université de Nice - Sophia Antipolis, F-06108 Nice Cedex 1, France \\ ${ }^{3}$ Cerro Tololo Inter-American Observatory, Casilla 603, La Serena, Chile
}

\section{Introduction}

Dome C, Antarctica is one of the most promising astronomical sites in the world (Fossat \& Candidi 2003, and references therein). Dome C boasts low wind speeds, very cold temperatures and little precipitation. The atmospheric turbulence is very weak compared to temperate sites, leading to sub-arcsecond seeing conditions (Lawrence et al. 2004; Agabi et al. 2006).

A Multi-Aperture Scintillation Sensor (MASS) was operated at Dome C $\left(123^{\circ} 21^{\prime \prime} \mathrm{E}\right.$, $75^{\circ} 06^{\prime \prime} \mathrm{S}, 3260 \mathrm{~m}$ ) during the first two months of the 2004 Antarctic winter season. The MASS instrument measures the scintillation of a single star; from this information the vertical distribution of atmospheric turbulence is derived. These data have been analysed in terms of seeing (Lawrence et al. 2004) and scintillation (Kenyon et al. 2006), here we summarise the main conclusions of the second paper and look at the implications for photometry and astrometry. The results are compared to similar data from Cerro Tololo $\left(70^{\circ} 48^{\prime \prime} \mathrm{W}, 30^{\circ} 09^{\prime \prime} \mathrm{S}, 2215 \mathrm{~m}\right)$ and Cerro Pachon $\left(70^{\circ} 44^{\prime \prime} \mathrm{W}, 30^{\circ} 14^{\prime \prime} \mathrm{S}, 2738 \mathrm{~m}\right)$ in Chile.

\section{Results}

A comparison of the turbulence profiles measured above Dome $\mathrm{C}$ with those of the two Chilean sites shows that Dome $\mathrm{C}$ has significantly less turbulence in all layers except the lowest layer. The most striking result is that the turbulence measured in the highest layer above Dome $\mathrm{C}$ is negligible compared to the Chilean sites. It is this high-altitude turbulence that has the largest influence on the astrometric and photometric precision achievable at a particular site. Using average wind speed profiles, we assess the photometric noise produced by scintillation, and the atmospheric contribution to the error budget in narrow angle differential astrometry.

\subsection{Photometry}

High-precision photometry is important, for example, for the detection of extra-solar planets and for observations of objects with very fast intensity changes (e.g., asteroseismology). The calculation of the photometric precision from atmospheric turbulence profiles depends on the length of the integration time and the size of the telescope. For the case of a long integration time on a large diameter telescope the photometric precision is expressed as

$$
\sigma_{I}=\left[10.7 \int h^{2} C_{n}^{2}(h) V^{-1}(h) \mathrm{d} h\right]^{1 / 2} D^{-2 / 3} t^{-1 / 2},
$$

where $h$ is the height above the site, $C_{n}^{2}$ is the refractive index structure constant, $V$ is the wind speed, $D$ is the telescope diameter and $t$ is the integration time. Fig. 1 (left) shows the median photometric precision at each site as a function of telescope diameter 

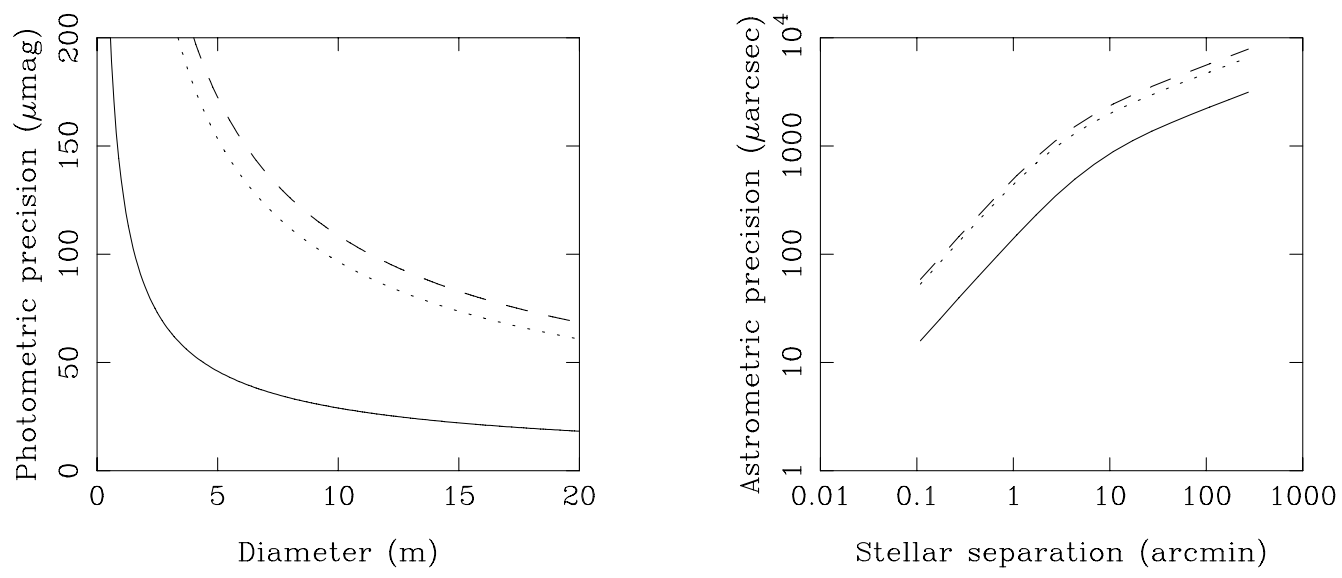

Figure 1. Left: the median photometric precision at Dome C (solid line), Cerro Tololo (dashed line) and Cerro Pachon (dotted line) as a function of telescope diameter, for a 60 s integration time. Right: the median astrometric precision at each site as a function of angular stellar separation, for a $1 \mathrm{hr}$ integration time and $10 \mathrm{~m}$ baseline.

for a $60 \mathrm{~s}$ integration. Dome $\mathrm{C}$ offers a gain of about 3.6 in photometric precision over the two Chilean sites.

\subsection{Astrometry}

Long baseline interferometry can be used to achieve high precision very narrow angle differential astrometry, benefiting science programs such as extrasolar planet searches and the study of close multiple star systems. Uncertainties in astrometric precision arise from instrumental effects and atmospheric effects. The astrometric uncertainty caused by the atmosphere can be calculated for two regimes, narrow-angle and very narrow angle, using the equations from Shao \& Colavita (1992).

$$
\sigma_{a t m}= \begin{cases}\theta^{1 / 3} t^{-1 / 2}\left[5.25 \int h^{2 / 3} C_{n}^{2}(h) V^{-1}(h) \mathrm{d} h\right]^{1 / 2} & \text { narrow angle, } \theta \bar{h} \gg B \\ \theta B^{-2 / 3} t^{-1 / 2}\left[5.25 \int h^{2} C_{n}^{2}(h) V^{-1}(h) \mathrm{d} h\right]^{1 / 2} & \text { very narrow angle, } \theta \bar{h} \ll B\end{cases}
$$

where, $B$ is the baseline length, $\theta$ is the angular separation between the two celestial objects and $\bar{h}$ is the turbulence weighted atmospheric height. Figure 1 (right) shows the median astrometric precision at each site as a function of angular separation for a $1 \mathrm{hr}$ integration and a $10 \mathrm{~m}$ baseline. Dome $\mathrm{C}$ offers a significant advantage in achievable astrometric precision.

\section{Conclusions}

Although the data from Dome $\mathrm{C}$ cover a fairly limited time frame, they lend strong support to expectations that Dome $\mathrm{C}$ will offer significant advantages for photometric and astrometric studies. Dome $\mathrm{C}$ offers a potential gain of about 3.6 in both long integration photometric precision and narrow-angle astrometry precision when compared to two midlatitude sites in Chile.

\section{References}

Agabi, A., Aristidi, E., Azouit, M., et al. 2006, PASP, 118, 344

Fossat, E., \& Candidi, M. (eds.) 2003, Mem. S.A.It. Supp., 2, 3

Kenyon, S. L., Lawrence, J. S., Ashley, M. C. B., et al. 2006, PASP, 118, 924

Lawrence, J. S., Ashley, M. C. B., Tokovinin, A., \& Travouillon, T. 2004, Nature, 431, 278

Shao, M., \& Colavita, M. M. 1992, A\&\&A, 262, 353 